\title{
Improved antitumor activity and reduced myocardial toxicity of doxorubicin encapsulated in MPEG-PCL nanoparticles
}

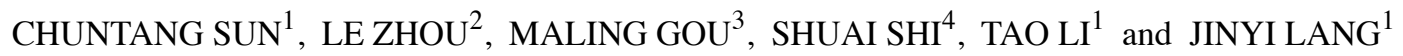 \\ ${ }^{1}$ Department of Radiation Oncology, Sichuan Cancer Hospital; ${ }^{2}$ Department of Health Management Center and \\ ${ }^{3}$ State Key Laboratory of Biotherapy and Cancer Center, West China Hospital, Sichuan University, Chengdu, Sichuan; \\ ${ }^{4}$ Institute of Biomedical Engineering, School of Ophthalmology and Optometry and Eye Hospital, \\ Wenzhou Medical University, Wenzhou, Zhejiang, P.R. China
}

Received December 29, 2015; Accepted February 11, 2016

DOI: $10.3892 /$ or.2016.4748

\begin{abstract}
Doxorubicin (Dox) is a broad-spectrum antitumor drug used for the treatment of many types of malignant tumors. Although it possesses powerful antitumor activity, its clinical application is seriously encumbered by its unselective distribution and systemic toxicities, particularly myocardial toxicity. Thus, it is imperative to modify Dox to decrease its systemic toxicities and improve its therapeutic index. In the present study, we adopted a novel type of monomethoxy poly(ethylene glycol)-poly( $\varepsilon$-caprolactone) (MPEG-PCL) micelles to encapsulate Dox to prepare Dox-loaded MPEG-PCL (Dox/MPEG-PCL) nanoparticles by a controllable self-assembly process. The cellular uptake efficiency and cell proliferation inhibition of the Dox/MPEG-PCL nanoparticles were examined. The antitumor activity of the Dox/ MPEG-PCL nanoparticles was tested on a multiple pulmonary metastasis model of melanoma on C57BL/6 mice. Systemic toxicities and survival time were compared between the mice treated with the Dox/MPEG-PCL nanoparticles and free Dox. The potential myocardial toxicity of the Dox/MPEG-PCL nanoparticles was investigated using a prolonged observation period. Encapsulation of Dox in MPEG-PCL nanoparticles significantly improved the cellular uptake and cell proliferation inhibition of Dox in vivo. Intravenous injection of Dox/ MPEG-PCL nanoparticles obtained significant inhibition of the growth and metastasis of melanoma in the lung and prolonged survival time compared with free Dox $(\mathrm{P}<0.05)$. The Dox/MPEG-PCL nanoparticles did not show obvious additional systemic toxicities compared with free Dox during the treatment time. During the prolonged observation period,
\end{abstract}

Correspondence to: Dr Chuntang Sun, Department of Radiation Oncology, Sichuan Cancer Hospital, 55 Renminnan Road, Sec. 4, Chengdu, Sichuan 610041, P.R. China

E-mail: sunrise14@163.com

Key words: drug delivery, MPEG-PCL, doxorubicin, nanoparticle, melanoma, pulmonary metastasis obvious decreased cardiac toxicity was observed in the Dox/ MPEG-PCL nanoparticle-treated mice compared with that observed in the free Dox-treated mice. These results indicated that encapsulating Dox with MPEG-PCL micelles could significantly promote its antitumor activity and reduce its toxicity to the myocardium.

\section{Introduction}

Although numerous new anticancer strategies have been developed during recent years, chemotherapy remains one of the most important means for the treatment of malignant tumors. Doxorubicin (Dox) is an anthracycline antibiotic isolated from Streptomyces peucetius. Due to its broad antitumor spectrum and effective antineoplastic activity, it has already been widely used in various hematologic malignancies and solid tumors, such as lymphoma (1), breast (2) and ovarian cancer (3), and soft tissue sarcomas (4). However, its clinical application is seriously constrained by its instability, unselective distribution and serious systemic toxicities, particularly dose-limiting myocardial toxicity, which could result in cardiomyopathy and congestive heart failure (5). These limit the clinical application and cumulative lifetime total dose of Dox (6). Hence, it is necessary to develop new strategies to decrease the toxicity and improve the therapeutic effect of Dox.

Among the various strategies to improve the therapeutic index of chemotherapy drugs, a drug delivery system based on biodegradable polymeric nanoparticles appears to be a promising direction which has attracted increased attention due to its superior cell uptake, passive targeting, improved anticancer efficiency and biological safety of the cargo (7).

The monomethoxy poly(ethylene glycol)-poly( $\varepsilon$ caprolactone) (MPEG-PCL) micelle is an amphiphilic, biocompatible and biodegradable copolymer nanoparticle. These features make MPEG-PCL micelles an ideal candidate for drug delivery system development. Previous studies have preliminarily showed the excellent drug loading capability of the MPEG-PCL micelle and its superior pharmacokinetics and drug release characteristics for drug encapsulation (8). These suggest that MPEG-PCL micelles may serve as a desired drug carrier for Dox. However, related research is rare. Only a few studies were limited to in vitro testing or subcutaneous tumor 
models treated with intratumoral injection. There are few systematic studies concerning its potential use by intravenous injection in lung metastasis models.

In the present study, we successfully prepared Dox-loaded MPEG-PCL (Dox/MPEG-PCL) nanoparticles using a selfassembly method. By encapsulating Dox in MPEG-PCL nanoparticles, Dox obtained superior cell uptake, improved anticancer effect and reduced toxicity to the myocardium compared with its free state. These results suggest a promising application of biodegradable MPEG-PCL nanoparticles to modify existing traditional chemotherapy drugs to decrease their systemic toxicities and improve their therapeutic index.

\section{Materials and methods}

Monomethoxy poly(ethylene glycol) (MPEG; Mn=2,000) was purchased from Sigma-Aldrich (St. Louis, MO, USA). $\varepsilon$-caprolactone $(\varepsilon-C L)$ was purchased from Alfa Aesar, USA. Dulbecco's modified Eagle's medium (DMEM) and 3-(4,5-dimethylthiazol-2-yl)-2,5-diphenyltetrazolium bromide (MTT) were both purchased from Sigma (St. Louis, MO, USA). Dox was purchased from Hisun Pharmaceutical Co. (Zhejiang, China). The lung carcinoma cell line A549 and the malignant melanoma cell line B16-F10 were purchased from the American Type Culture Collection (ATCC; Manassas, VA, USA). The cells were incubated in a $95 \%$ air-humidified atmosphere containing $5 \% \mathrm{CO}_{2}$ at $37^{\circ} \mathrm{C}$. The female $\mathrm{C} 57 \mathrm{BL} / 6$ mice (6-8 weeks old) were purchased from the Experimental Animal Center of Sichuan University (Chengdu, Sichuan, China). The mice were keep at room temperature with a humidity of $50-60 \%$. All animal care and experimental procedures in the present study were conducted according to institutional animal care and use guidelines.

Preparation of Dox-loaded MPEG-PCL nanoparticles. Dox-loaded MPEG-PCL (Dox/MPEG-PCL) nanoparticles were synthesized by a self-assembly process according to previous studies $(8,9)$. In brief, empty MPEG-PCL micelles without encapsulated drug were initially prepared by a selfassembly procedure triggered by heat rise. Firstly, MPEG-PCL diblock copolymer was fully dissolved in cold water. Then, the temperature of the MPEG-PCL solution was increased to $50^{\circ} \mathrm{C}$. Due to the amphiphilic property of its molecular structure, the MPEG-PCL may automatically assemble into nano-sized micelles (with the PCL segment formed the hydrophobic core of the micelle and PEG segment became the hydrophilic shell of the micelle) triggered by a temperature increase in the water solution under moderate stirring. After the blank MPEG-PCL micelles were prepared, the Dox/MPEG-PCL nanoparticles were prepared by a $\mathrm{pH}$ sensitive self-assembly process. In short, Dox dissolved in water was slowly added into the blank MPEG-PCL micelles in phosphate-buffered saline (PBS) ( $\mathrm{pH}$ 7.4) drop by drop. Due to the sudden change in the low solubility of Dox at pH 7.4, Dox was encapsulated into the hydrophobic core of the MPEG-PCL micelles. Finally, the Dox/MPEG-PCL nanoparticles were purified and lyophilized for future use.

Physicochemical property and morphologic characterization. Dox was successfully incorporated into the MPEG-PCL nanoparticles. The basic characteristics of particle size and zeta potential of the Dox/MPEG-PCL nanoparticles was determined by Nano ZS90 (Malvern Instruments, UK) at room temperature. The ultra microstructure of the Dox/MPEG-PCL nanoparticles was observed by a transmission electron microscope (TEM) (H-6009IV; Hitachi, Tokyo, Japan).

Cellular uptake in vitro and in vivo. As a model drug, one important feature of Dox which makes it easily detectable is its fluorescence. Dox-derived fluorescence can be excited at a 488-nm wavelength and detected at a 564-nm wavelength (red fluorescence). In order to compare the difference in cellular uptake between free Dox and Dox/MPEG-PCL nanoparticles, fluorescence microscopy was adopted. Briefly, A549 cells were plated on a coverglass into 6 -well plates at a density of $1 \times 10^{5}$ and incubated overnight. Then, the cells were treated with free Dox or Dox/MPEG-PCL nanoparticles, respectively. After co-incubation for $6 \mathrm{~h}$, the cells on the coverglass were taken out for detection under a fluorescence microscope. Before observation under a fluorescence microscope, cell nuclei were pre-stained with DAPI. For in vivo cellular uptake, $2 \times 10^{4}$ B16-F10 cells were injected into a zebra fish $72 \mathrm{~h}$ post fertilization (hpf), and 2 days later, Dox/MPEG-PCL nanoparticles were administered by micro-injection. Twenty-four hours later, the fish were observed under a fluorescence microscope.

In vitro antitumor effect. The in vitro antitumor effect of the Dox/MPEG-PCL nanoparticles was determined by MTT assay in B16-F10 cells. Briefly, the cells were plated in 96-well plates at a density of $2 \times 10^{3}$ cells/well in $100 \mu \mathrm{l}$ DMEM and grown overnight. Then, $100 \mu 1$ DMEM containing a series of ascending concentrations of free Dox or Dox/MPEG-PCL nanoparticles were added to each well, respectively. Each concentration was set with 3 duplicate wells. Untreated cells were used as the control. After incubation for $48 \mathrm{~h}, 20 \mu \mathrm{l}$ of MTT solution $(5 \mathrm{mg} / \mathrm{ml})$ was added to each well, and then incubated at $37^{\circ} \mathrm{C}$ for $4 \mathrm{~h}$. Then, MTT solution was removed and $150 \mu \mathrm{l}$ DMSO was added to each well. Absorbance of the formazan crystals was detected at $570 \mathrm{~nm}$. Cell viability was compared with the control cells and calculated as a percentage.

In vivo antitumor effect. In order to verify the in vivo anticancer effect and investigate the possible mechanism of Dox/MPEG-PCL nanoparticles, we tested the Dox/ MPEG-PCL nanoparticles on a highly malignant multiple pulmonary metastasis model of melanoma. To generate the multiple pulmonary metastatic model of melanoma, $1.5 \times 10^{5}$ B16-F10 cells fully resuspended in $0.2 \mathrm{ml}$ of normal saline (NS) were intravenously injected into the lateral dorsal tail vein of C57BL/6 mice. Five days after inoculation, the mice were randomly divided into 4 groups (6 mice/group, with an average body weight of $20 \mathrm{~g}$ ) and treated with an intravenous injection of the following regimens, respectively; i) $100 \mu \mathrm{l}$ of PBS; ii) $2 \mathrm{mg}$ of blank MPEG-PCL in $100 \mu \mathrm{l}$ PBS; iii) $0.1 \mathrm{mg}$ free Dox in $100 \mu \mathrm{l}$ PBS (Dox dose, $5 \mathrm{mg} / \mathrm{kg}$ ); and iv) $0.1 \mathrm{mg}$ Dox encapsulated in $2 \mathrm{mg}$ MPEG-PCL in $100 \mu \mathrm{l}$ PBS (Dox dose, $5 \mathrm{mg} / \mathrm{kg}$ ). The administrations were repeated twice a week for a total number of 6 times. Three days after the last time of treatment, the mice were sacrificed, and both lungs of every mouse were dissected for further evaluation. Since 
metastatic lesions of B16-F10 melanoma are black or brown they are readily visually detected. The numbers of surface metastatic lesions of each mice were counted under a stereoscopic microscope (magnification, $\mathrm{x} 10$ ) by two independent investigators who were blinded to the group assignment. The metastasis index of each mice was defined as the sum of scores of all visible metastatic lesions in both lungs as previously detailed: metastatic lesions $\leq 1 \mathrm{~mm}$ were scored as 1 , metastatic lesions $>1 \mathrm{~mm}$ but $\leq 2 \mathrm{~mm}$ were scored as 3 , and lesions $>2 \mathrm{~mm}$ were scored as 10 (10). In the survival analysis, the experiment was repeated, and the observation period was extended to as long as 50 days after inoculation.

Systemic toxicity evaluation. To detect the possible toxicities of the Dox/MPEG-PCL nanoparticles, at the end of the in vivo experiment, major organs (heart, liver, spleen, lung and kidney) of each group were also harvested at the same time. Paraffin-embedded sections of the above organs were stained with hematoxylin and eosin (H\&E) method. The toxicities that induced pathological changes to the major organs were observed under a microscope by two experienced pathologists in a blinded manner. Since the myocardial toxicity of Dox is a dose accumulated toxicity and usually occur at a period of time after treatment, it is usually difficult for the mice to survive over 30 days once inoculated with B16-F10 cells. Thus, in order to reveal the potential myocardial protection of the Dox/MPEG-PCL nanoparticles, we repeated the treatment regimens on two groups of mice without tumors and fed them as long as 10 weeks after the last intravenous administration.

Statistical analyses. All data are expressed as mean \pm SD. Each experimental group contained 6 mice. Comparison of the numbers of metastatic lesions and the metastasis index between each group was performed by analysis of variance (ANOVA). For survival analysis, data were analyzed using the Kaplan-Meier method. Statistical analysis was carried out using Prism GraphPad software and SPSS statistical software (version 22.0; SPSS, Inc., IBM Co., Armonk, NY, USA). P <0.05 was considered to indicate a statistically significant result.

\section{Results}

Characterization of the Dox-loaded MPEG-PCL nanoparticles. The Dox/MPEG-PCL nanoparticles were easily prepared by simple heat triggering and then a pH-sensitive self-assembly method as mentioned above. The process is illustrated as a sketch map in Fig. 1A. Briefly, blank MPEG-PCL micelles without Dox were first prepared ahead using a heat-induced self-assembly process at $50^{\circ} \mathrm{C}$, and then Dox was encapsulated into blank MPEG-PCL micelles by a rapid $\mathrm{pH}$-sensitive automatically self-assembly process under moderate stirring. The technological requirements for the whole process are simple, safe and mild, without introducing any unwanted organic solvents and surfactants. The whole procedure did not require any extreme conditions or complicated physicochemical process. All the above features enable the preparation of Dox/MPEG-PCL nanoparticles to be easily scaled up for mass production.

For characterization of the Dox/MPEG-PCL nanoparticles, we used Zetasizer Nano Z90 (Malvern Instruments) to determine the particle size and zeta potential. As shown in Fig. 1B, the Dox/MPEG-PCL nanoparticles we prepared were relatively neat and uniform, with a mean particle size of $\sim 41.9 \mathrm{~nm}$. Its zeta potential was $-5.6 \mathrm{mV}$ at $\mathrm{pH} 7.0$. Under transmission electron microscopy (Fig. 1C), Dox/MPEG-PCL nanoparticles showed classical spherical shape (mean diameter $\sim 35 \mathrm{~nm}$ ) and at a mono-dispersed state did not form severe aggregation. Severe aggregation results in pulmonary embolism and is dangerous for intravenous administration. By repeated examinations, the MPEG-PCL had an average drugloading content [the ratio of incorporated drug to polymer $(w / w)]$ of $5.33 \pm 0.54$ and an entrapment efficiency as high as 94\% for Dox at room temperature.

Increased cellular uptake. In order to compare the cellular uptake between free Dox and Dox/MPEG-PCL, we treated B16-F10 cells with Dox and the Dox/MPEG-PCL nanoparticles, respectively. Dox is a fluorescent substance. Thus, to exhibit the intake and location of Dox, we used fluorescence microscopy to capture the distribution of Dox. As shown in Fig. 2A-a, in the free Dox-treated cells, the red fluorescence of Dox was scattered around the cells, without any obvious aggregation tendency around the nucleus. In contrast, in the Dox/MPEG-PCL nanoparticle-treated cells (2A-b), the red fluorescence of Dox was significantly gathered around the nucleus, demonstrating a distinct intracellular accumulation trend. In vivo, we also preliminarily observed the relative enrichment of the Dox/MPEG-PCL nanoparticles at the tumor cell injection site in the zebra fish model (2A-c). These results indicated that the cellular uptake of the Dox/MPEG-PCL nanoparticles was obvious more effective than that of free Dox.

Enhanced anticancer activity in vitro. We further investigated the cell proliferation inhibition of the Dox/MPEG-PCL nanoparticles using MTT assay. Inhibition of the proliferation of B16-F10 cells by Dox and the Dox/MPEG-PCL nanoparticles is shown in Fig. 2B. The results showed that treatment with the Dox/MPEG-PCL nanoparticles resulted in a significantly enhanced inhibition of tumor cell proliferation compared with free Dox at equal equivalent concentrations of Dox. Notably, this phenomenon was relatively more obvious at a low equivalent concentration of Dox. When the equivalent concentration of Dox increase above $5 \mu \mathrm{g} / \mathrm{ml}$, the discrepancy was not obvious. This result indicated that the Dox/MPEG-PCL nanoparticles could significantly enhance the antitumor effect of Dox compared with its free state in vitro.

Improved in vivo antitumor efficiency and prolonged survival. To investigate the antitumor efficiency and potential underlying mechanism of the Dox/MPEG-PCL nanoparticles, a multiple pulmonary metastasis model of B16-F10 cells in C57BL/6 mice was established. The mice were then treated with an intravenously injection of NS, blank MPEG-PCL, free Dox or Dox/MPEG-PCL, respectively. As shown in Fig. 2C, although free Dox and Dox/MPEG-PCL nanoparticle-treated mice both showed obviously suppressed tumor growth compared with the mice treated with NS or blank MPEG-PCL nanoparticles, the Dox/MPEG-PCL nanoparticle-treated mice exhibited less and smaller metastatic nodules compared with that observed 
A

blank MPEG-PCL micelles
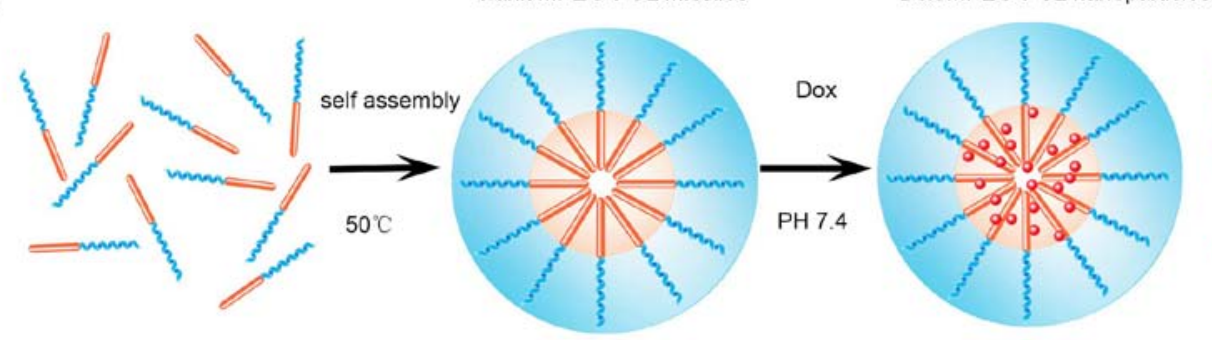

Dox/MPEG-PCL nanoparticles

B

C
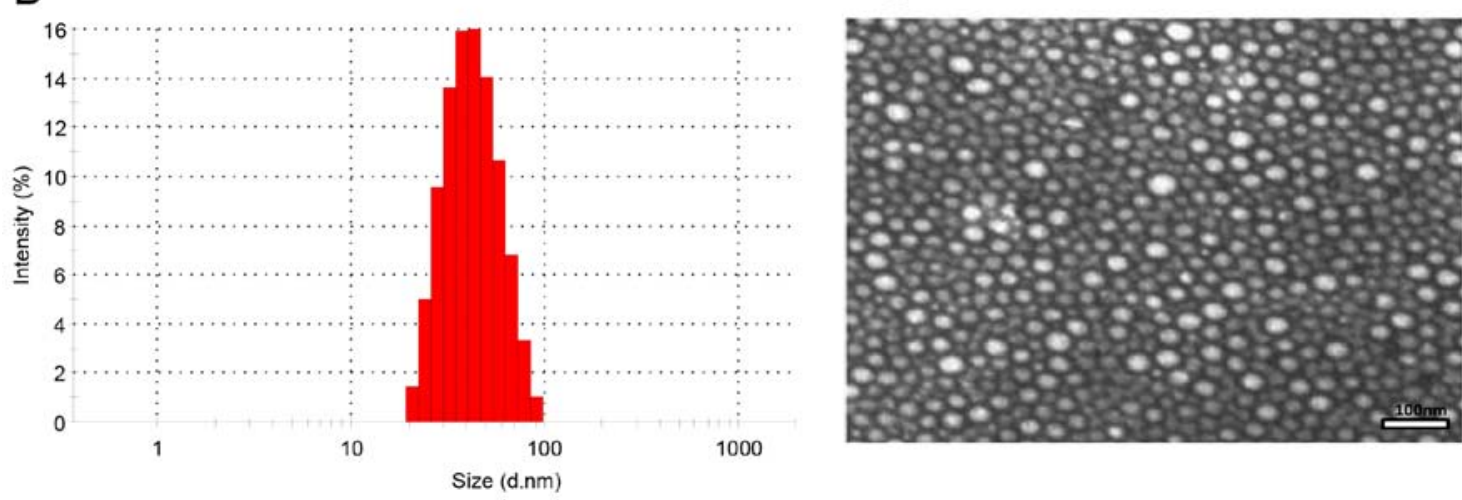

Figure 1. (A) Condensed schematic diagram of the preparation process of the Dox/MPEG-PCL nanoparticles. (B) Particle size distribution of Dox/MPEG-PCL nanoparticles. (C) Dox/MPEG-PCL nanoparticles under TEM.

A

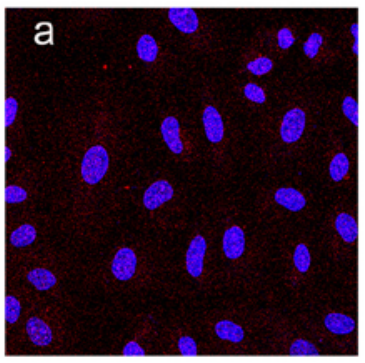

Free Dox

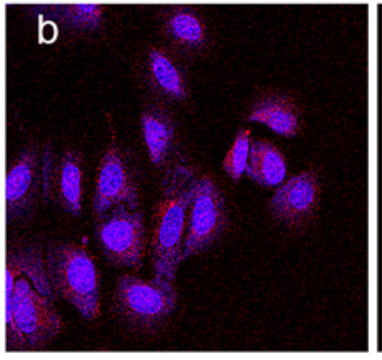

DOX/MPEG-PCL

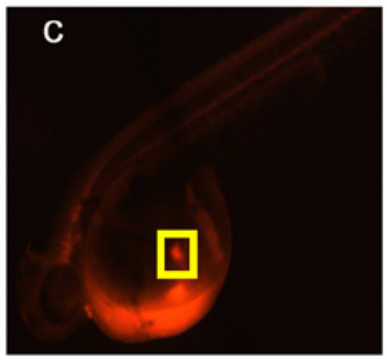

Dox/MPEG-PCL in zebra fish

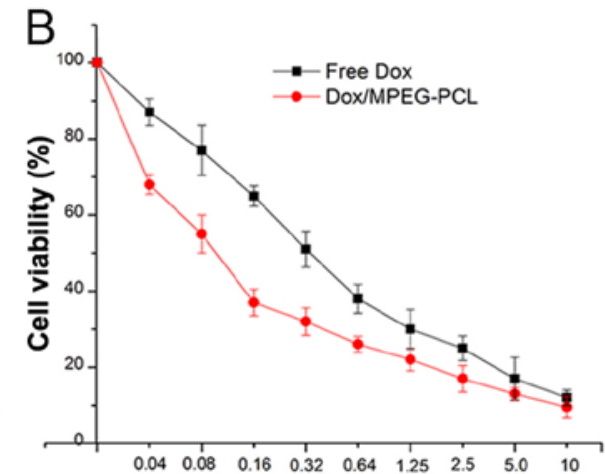

Dox concentration $(\mu \mathrm{g} / \mathrm{ml})$

C

a

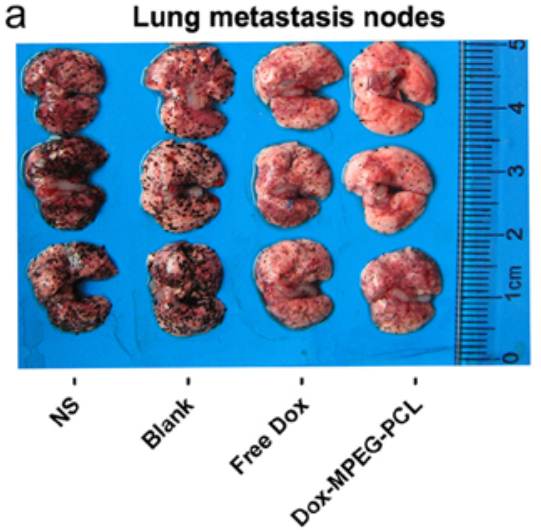

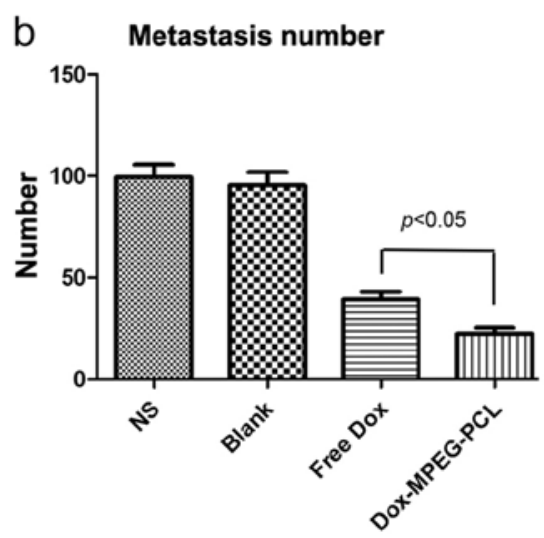

C

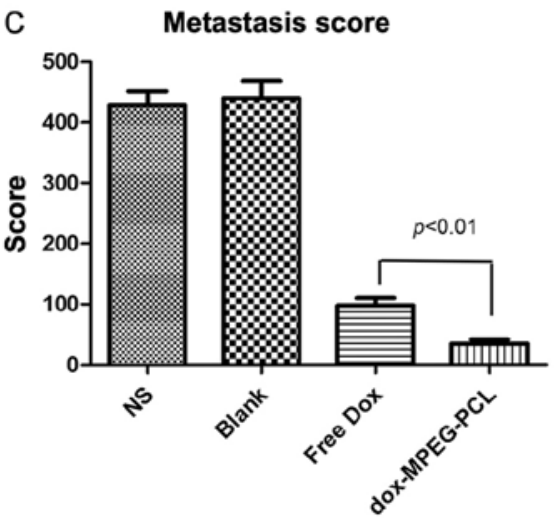

Figure 2. (A-a) Cells treated with free Dox. (b) Cells treated with the Dox/MPEG-PCL nanoparticles. Cell nuclei were pre-stained with DAPI. Images were fused by two channels of Dox-derived red fluorescence and DAPI-derived blue fluorescence. (c) Relative enrichment of Dox/MPEG-PCL nonoparticles focused at the tumor cell injection site (in the yellow rectangle) on a zebra fish. (B) Inhibition of cell proliferation of the Dox/MPEG-PCL nanoparticles determined by the MTT method. Data are expressed as percentages relative to control cells (treated with medium only). All data are expressed as means \pm SD, $n=3$. (C-a) Representative image of lungs bearing metastatic nodules of B16-F10 melanoma in each group. (b) Evaluation of the numbers of the metastatic nodules on the lung surface of each group. The number of metastatic nodules was significantly reduced in the Dox/MPEG-PCL group than the number of nodules in the free Dox group $(\mathrm{P}<0.05)$. (c) Evaluation of the metastasis index based on the number and diameter of the metastatic nodules. The index was significantly lower in the Dox/MPEG-PCL-treated mice than that in the free Dox-treated mice $(\mathrm{P}<0.01)$. 
A

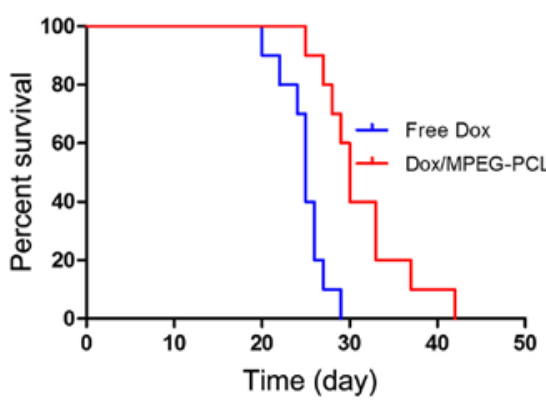

B

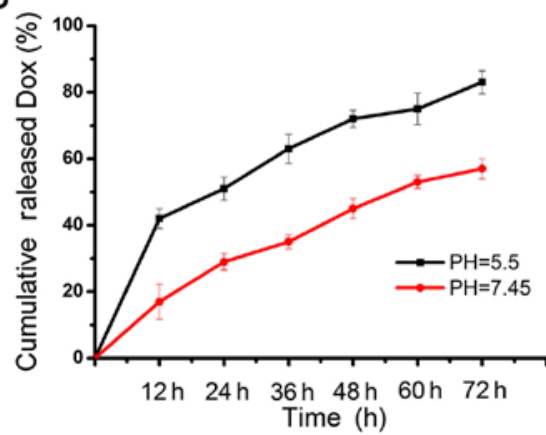

Figure 3. (A) Kaplan-Meier survival curve for tumor-bearing mice treated with free Dox and Dox/MPEG-PCL nanoparticles. Survival time was significantly longer in the Dox/MPEG-PCL nanoparticle-treated mice compared with the survival time in the free Dox-treated mice $(\mathrm{P}<0.05)$. $(\mathrm{B}) \mathrm{Dox}$ release profile of Dox/MPEG-PCL nanoparticles under different $\mathrm{pH}$ conditions.

A
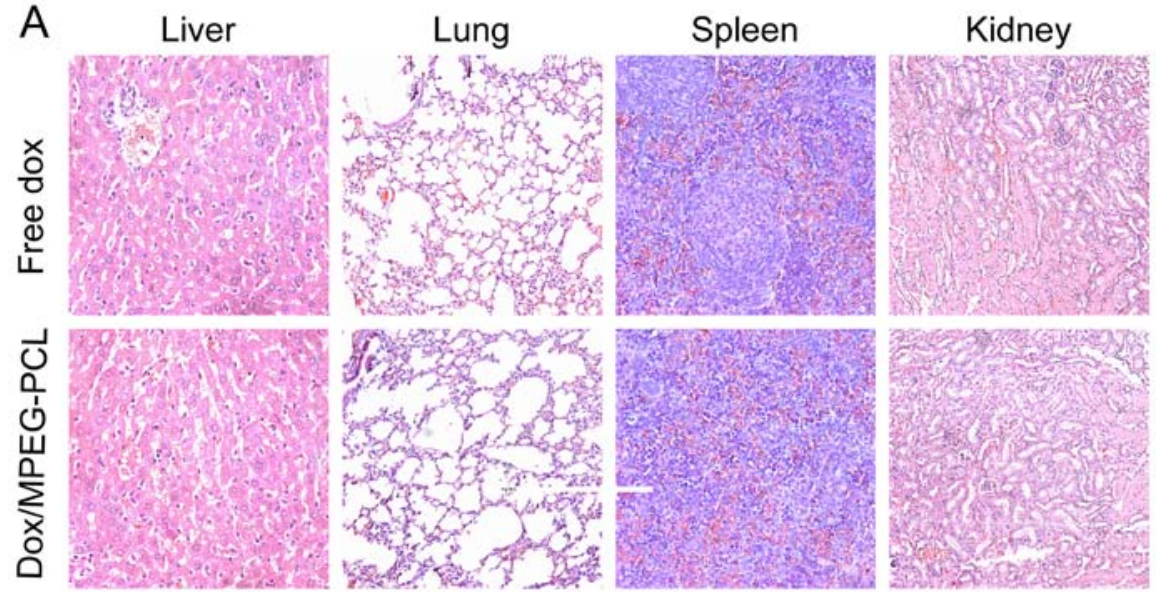

B

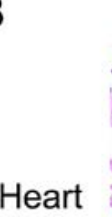

Normal
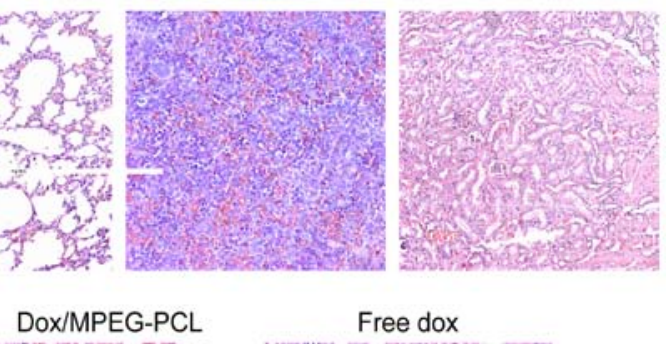

Free dox

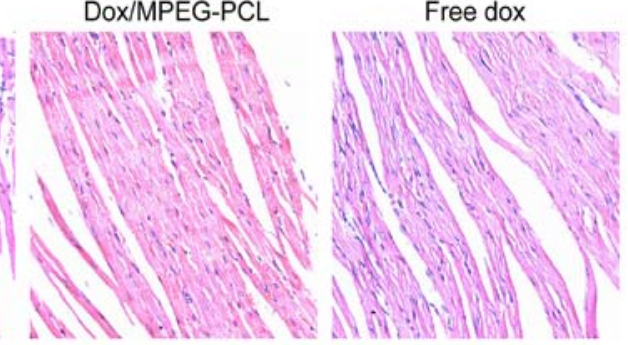

Figure 4. (A) H\&E staining of the major organs of mice treated with NS, blank MPEG-PCL nanoparticles, free Dox and Dox/MPEG-PCL nanoparticles, respectively. (B) H\&E staining of cardiac muscle sections from tumor-free mice receiving the same treatment scheme as previously outlined. Hearts were harvested 10 weeks after treatment.

in the mice treated with free Dox (Fig. 2C-a). To compare the difference, we counted the number of metastatic nodules for each mouse and constructed a metastasis index based on the number and diameter of the metastatic nodules according to previous literature (11). As shown in Fig. 2C-b, the Dox/ MPEG-PCL nanoparticle-treated mice exhibited significantly reduced surface metastatic nodules compared with the number of nodules in the mice treated with free Dox $(\mathrm{P}<0.05)$. After we scored the metastatic nodules by size (Fig. 2C-c), the improved antitumor effect represented in the metastasis index was even more significant $(\mathrm{P}<0.01)$. These findings indicated that compared with its free state, Dox-loaded MPEG-PCL nanoparticles did not only reduce tumor lung metastasis but also inhibited the growth of lung metastases.
Survival analysis. In order to explore the potential benefit of Dox/MPEG-PCL nanoparticles in terms of survival, a survival study was carried out. The results indicated that, although the Dox/MPEG-PCL nanoparticle- and free Dox-treated mice generally lived obviously longer than the NS or blank MPEG-PCL-treated mice, the mice treated with the Dox/ MPEG-PCL nanoparticles exhibited significantly prolonged survival time $(\mathrm{P}<0.05)$ when compared with that of free Dox-treated mice as shown in the survival curve in Fig. 3A. There was no significant difference in survival between the NS-treated and blank MPEG-PCL-treated mice ( $P>0.05)$. Therefore, this result indicated that the encapsulation of Dox into MPEG-PCL nanoparticles did not only improve inhibition of the growth of lung metastasis, but also significantly 
prolonged the overall survival time when compared with free Dox.

Comparison of the systemic toxicity. In order to appraise the toxicity of the Dox/MPEG-PCL nanoparticles, we monitored the body weight and other general conditions such as appetite, weight loss and behavior change during the entire treatment period. Compared with the mice treated with free Dox, the mice treated with the Dox/MPEG-PCL nanoparticles showed an overall better general condition and relative less weight loss, but there was no significant difference $(\mathrm{P}>0.05)$. After the mice were sacrificed, their main organs were harvested for H\&E staining. As shown in Fig. 4A, there were no obvious histopathologic difference in the main organs between the free Dox- and Dox/MPEG-PCL nanoparticle-treated mice. This demonstrated that, when compared with free Dox, Dox-loaded in MPEG-PCL nanoparticles at least did not introduce additional acute systemic toxicity during treatment.

Comparison of chronic myocardial toxicity. Chronic myocardial toxicity is the dose-limiting toxicity of Dox which is closely related to the accumulation dose. It usually appears in a period of time after treatment. In order to assess the chronic myocardial toxicity of Dox, we repeated the experiment on mice without tumors and extended the observation time to 10 weeks after the last time of administration. As shown in Fig. 4B, the heart tissue sections from free Dox-treated mice exhibited serious myocardial pathological changes; the myocardial fibers became thin and loose, and even presented varying degrees of rupture. However, the heart tissue sections from the Dox/MPEG-PCL nanoparticle-treated mice only showed slightly thin myocardial fibers compared with the normal muscle fibers. In comarison with free Dox, this indicated that Dox/MPEG-PCL nanoparticles significantly reduced the chronic myocardial toxicity of Dox during the period of treatment.

\section{Discussion}

Although traditional chemotherapeutic agents have made great progress in the treatment of cancer in recent decades, further progress is limited due to inherent shortcomings, including serious systemic toxicities and non-specific distribution (12). Thus, further research to modify these agents in order to alleviate their toxicities and obtain better treatment efficacy is urgently needed.

The clinical use of Dox, as one of the most widely used antitumor drugs, is obstructed by its serious systemic toxicities particularly cardiac toxicity and limited lifetime dose (13). In order to overcome these shortcomings, various drug delivery systems have been developed such as liposome $(14,15)$ and chitosan nanoparticles $(16,17)$, super paramagnetic iron oxide nanoparticles (SPIO) (18) and silica nanoparticles (19). However, their particle sizes usually range from 150 to $800 \mathrm{~nm}$, which are likely grasped by the spleen and removed by phagocytes when injected intravenously (20). In addition, various types of these nanoparticles are prepared using an emulsion solvent extract method, which may introduce undesirable organic solvents and surfactants to the products $(21,22)$. Furthermore, various inorganic nanoparticles are not biocompatible and biodegradable thus may result in serious consequences even death when intravenously used (23).

Recently, block copolymer nanoparticles have emerging as a new promising drug delivery system and has attracted much attention (24). In the present study, we employed amphiphilic and biodegradable MPEG-PCL diblock copolymer nanoparticles as a drug carrier and successfully encapsulated Dox to prepare Dox-loaded MPEG-PCL nanoparticles. Unlike the preparation of many other drug-loaded polymeric nanoparticles, we prepared Dox/MPEG-PCL by a self-assembly method rather than an emulsion solvent extract method which may produce larger size nanoparticles than we wanted and introduce unwanted organic solvents, surfactants difficult to remove. The prepared nanoparticles had a desired average particle size of $\sim 42 \mathrm{~nm}$, and superior solubility after freeze drying.

As a drug delivery system, the particle size is very important (25). It has been identified that particles larger than $200 \mathrm{~nm}$ usually are grasped by the spleen and removed by phagocytes when intravenously injected. On the contrary, particles $<20 \mathrm{~nm}$ are quickly removed through renal clearance $(26,27)$. Based on the above findings, there is a consensus that particle sizes ranging from 20 to $100 \mathrm{~nm}$ are ideal for intravenous administration that may acquire extended halflife in the bloodstream (28). Moreover, new vessels in tumors are more leaky due to abnormal endothelial cells with large spaces, ranging from $200 \mathrm{~nm}$ to $1.2 \mu \mathrm{m}$. Particles within this range would easily extravasate from the new vessels around tumor tissue, and thus could selectively increase the drug accumulation at the tumor site through enhanced permeation and retention (EPR) effect (29). In the present study, we prepared the Dox/MPEG-PCL nanoparticles with a desired particle size $(\sim 42 \mathrm{~nm})$, and thus enhanced the selective distribution of the drug at the tumor site by the EPR effect.

In addition to particle size, zeta potential may be another important factor that contributes to the passive targeting effect of the Dox/MPEG-PCL nanoparticles. The Dox/MPEG-PCL nanoparticles had a slightly negative zeta potential $\sim-5.6 \mathrm{mV}$. When intravenously injected, the negative zeta potential rejected the plasma protein with a negative charge thus increasing the plasmatic circulation time and the probability for Dox to access the tumor site (30).

When Dox/MPEG-PCL nanoparticles arrive at the tumor site, low $\mathrm{pH}$ may change the zeta potential in a positive direction. This may enhance its binding with the negative charge of the cell membrane thus increasing the cellular uptake of Dox (31). In addition, $\mathrm{pH}$ is also a critical factor that could affect Dox drug release. As known, the $\mathrm{pH}$ in tumor tissues is usually lower due to hypoxia and accumulation of lactic acid. Previous research and our data both showed that the Dox release process from Dox/MPEG-PCL nanoparticles is a $\mathrm{pH}$-dependent process which is more rapid at $\mathrm{pH} 5.5$ than at pH 7.45 (32) (Fig. 3B). When Dox/MPEG-PCL nanoparticles arrive at the tumor site, low $\mathrm{pH}$ may trigger the quick release of the Dox encapsulated in the MPEG-PCL micelles and facilitate the passive accumulation of Dox at the tumor site, thus eventually resulting in a higher local concentration than free Dox.

In the present study, we confirmed the significant increase in cellular uptake and enhanced proliferation inhibition of 
tumor cells treated with the Dox-encapsulated MPEG-PCL nanoparticles in vitro. In vivo, the multiple pulmonary metastasis model of melanoma further demonstrated the improved antitumor effect and obviously decreased myocardial toxicity of the Dox/MPEG-PCL nanoparticles than these parameters noted for free DOX. Based on the main characteristics of the Dox/MPEG-PCL nanoparticles and the above analysis, we may deduce that the desired particle size, slightly negative zeta potential, pH-dependent release and EPR effect all contribute to the superior antitumor activity and attenuation of the toxicity of the Dox/MPEG-PCL nanoparticles. Considering the advantages and safety, Dox/MPEG-PCL nanoparticles may serve as a new approach for tumor treatment, and encapsulation of drugs in MPEG-PCL nanoparticles may represent a promising strategy for traditional drug modification.

\section{Acknowledgements}

This study was supported by Science Foundation of Chengdu Municipal Bureau of Science and Technology, Grant No. 2015HM01-00254-SF.

\section{References}

1. Azim HA, Santoro L, Bociek RG, Gandini S, Malek RA and Azim HA Jr: High dose intensity doxorubicin in aggressive nonHodgkin's lymphoma: A literature-based meta-analysis. Ann Oncol 21: 1064-1071, 2010.

2. Sauter C: Doxorubicin adjuvant combinations for breast cancer. Lancet 342: 1550-1551, 1993.

3. Staropoli N, Ciliberto D, Botta C, Fiorillo L, Grimaldi A, Lama S, Caraglia M, Salvino A, Tassone P and Tagliaferri P: Pegylated liposomal doxorubicin in the management of ovarian cancer: A systematic review and metaanalysis of randomized trials. Cancer Biol Ther 15: 707-720, 2014.

4. Grenader T, Goldberg A, Hadas-Halperin I and Gabizon A: Long-term response to pegylated liposomal doxorubicin in patients with metastatic soft tissue sarcomas. Anticancer Drugs 20: 15-20, 2009.

5. Heger Z, Cernei N, Kudr J, Gumulec J, Blazkova I, Zitka O, Eckschlager T, Stiborova M, Adam V and Kizek R: A novel insight into the cardiotoxicity of antineoplastic drug doxorubicin. Int J Mol Sci 14: 21629-21646, 2013.

6. Chatterjee K, Zhang J, Honbo N and Karliner JS: Doxorubicin cardiomyopathy. Cardiology 115: 155-162, 2010.

7. Wright J: Nanotechnology: Deliver on a promise. Nature 509: S58-S59, 2014.

8. Gou M, Zheng X, Men K, Zhang J, Wang B, Lv L, Wang X, Zhao Y, Luo F, Chen L, et al: Self-assembled hydrophobic honokiol loaded MPEG-PCL diblock copolymer micelles. Pharm Res 26: 2164-2173, 2009.

9. Zheng L, Gou M, Zhou S, Yi T, Zhong Q, Li Z, He X, Chen X, Zhou L, Wei Y, et al: Antitumor activity of monomethoxy poly(ethylene glycol)-poly( $\varepsilon$-caprolactone) micelle-encapsulated doxorubicin against mouse melanoma. Oncol Rep 25: 1557-1564, 2011.

10. van Spriel AB, van Ojik HH, Bakker A, Jansen MJ and van de Winkel JG: Mac-1 (CD11b/CD18) is crucial for effective Fc receptor-mediated immunity to melanoma. Blood 101: 253-258, 2003

11. Tsai NM, Chen BM, Wei SL, Wu CW and Roffler SR: Anti-tumor immunoglobulin $\mathrm{M}$ increases lung metastasis in an experimental model of malignant melanoma. Clin Exp Metastasis 20: 103-109, 2003.

12. Bourzac K: Nanotechnology: Carrying drugs. Nature 491: S58-S60, 2012.

13. Aiken MJ, Suhag V, Garcia CA, Acio E, Moreau S, Priebat DA, Chennupati SP and Van Nostrand D: Doxorubicin-induced cardiac toxicity and cardiac rest gated blood pool imaging. Clin Nucl Med 34: 762-767, 2009.
14. Liu Y, Fang J, Kim YJ, Wong MK and Wang P: Codelivery of doxorubicin and paclitaxel by cross-linked multilamellar liposome enables synergistic antitumor activity. Mol Pharm 11: 1651-1661, 2014.

15. Green AE and Rose PG: Pegylated liposomal doxorubicin in ovarian cancer. Int J Nanomedicine 1: 229-239, 2006.

16. Unsoy G, Khodadust R, Yalcin S, Mutlu P and Gunduz U: Synthesis of Doxorubicin loaded magnetic chitosan nanoparticles for $\mathrm{pH}$ responsive targeted drug delivery. Eur J Pharm Sci 62: 243-250, 2014.

17. Sanyakamdhorn S, Agudelo D and Tajmir-Riahi HA: Encapsulation of antitumor drug Doxorubicin and its analogue by chitosan nanoparticles. Biomacromolecules 14: 557-563, 2013.

18. Yang X, Grailer JJ, Rowland IJ, Javadi A, Hurley SA, Steeber DA and Gong S: Multifunctional SPIO/DOX-loaded wormlike polymer vesicles for cancer therapy and MR imaging. Biomaterials 31: 9065-9073, 2010.

19. Meng H, Liong M, Xia T, Li Z, Ji Z, Zink JI and Nel AE: Engineered design of mesoporous silica nanoparticles to deliver doxorubicin and P-glycoprotein siRNA to overcome drug resistance in a cancer cell line. ACS Nano 4: 4539-4550, 2010.

20. Jia Y, Yuan M, Yuan H, Huang X, Sui X, Cui X, Tang F, Peng J, Chen J, Lu S, et al: Co-encapsulation of magnetic $\mathrm{Fe}_{3} \mathrm{O}_{4}$ nanoparticles and doxorubicin into biodegradable PLGA nanocarriers for intratumoral drug delivery. Int J Nanomedicine 7: 1697-1708, 2012.

21. Gaucher G, Dufresne MH, Sant VP, Kang N, Maysinger D and Leroux JC: Block copolymer micelles: Preparation, characterization and application in drug delivery. J Control Release 109: $169-188,2005$

22. Farokhzad OC, Jon S, Khademhosseini A, Tran TN, Lavan DA and Langer R: Nanoparticle-aptamer bioconjugates: A new approach for targeting prostate cancer cells. Cancer Res 64: 7668-7672, 2004

23. Hudson SP, Padera RF, Langer R and Kohane DS: The biocompatibility of mesoporous silicates. Biomaterials 29: 4045-4055, 2008.

24. Chow EK, Zhang XQ, Chen M, Lam R, Robinson E, Huang H, Schaffer D, Osawa E, Goga A and Ho D: Nanodiamond therapeutic delivery agents mediate enhanced chemoresistant tumor treatment. Sci Transl Med 3: 73ra21, 2011.

25. Canelas DA, Herlihy KP and DeSimone JM: Top-down particle fabrication: Control of size and shape for diagnostic imaging and drug delivery. Wiley Interdiscip Rev Nanomed Nanobiotechnol 1: 391-404, 2009.

26. Gupta AK and Gupta M: Synthesis and surface engineering of iron oxide nanoparticles for biomedical applications. Biomaterials 26: 3995-4021, 2005.

27. Gupta AK and Wells S: Surface-modified superparamagnetic nanoparticles for drug delivery: Preparation, characterization, and cytotoxicity studies. IEEE Trans Nanobioscience 3: 66-73, 2004.

28. Agarwal A, Mackey MA, El-Sayed MA and Bellamkonda RV: Remote triggered release of doxorubicin in tumors by synergistic application of thermosensitive liposomes and gold nanorods. ACS Nano 5: 4919-4926, 2011.

29. Matsumura $Y$ and Maeda H: A new concept for macromolecular therapeutics in cancer chemotherapy: Mechanism of tumoritropic accumulation of proteins and the antitumor agent smancs. Cancer Res 46: 6387-6392, 1986.

30. Sant S, Poulin S and Hildgen P: Effect of polymer architecture on surface properties, plasma protein adsorption, and cellular interactions of pegylated nanoparticles. J Biomed Mater Res A 87: 885-895, 2008

31. Lu J, Jia H, Guo L, Zhang G, Cao Y, Yan H and Liu K: Zwitterionic polymeric micelles that undergo a $\mathrm{pH}$-triggered positive charge for enhanced cellular uptake. Eur Polym J 66: 376-385, 2015.

32. Gou M, Zheng X, Men K, Zhang J, Zheng L, Wang X, Luo F, Zhao Y, Zhao X, Wei Y, et al: Poly(epsilon-caprolactone)/ poly(ethylene glycol)/poly(epsilon-caprolactone) nanoparticles: Preparation, characterization, and application in doxorubicin delivery. J Phys Chem B 113: 12928-12933, 2009. 